\title{
Evaluación de tratamientos para la propagación de ceiba barrigona (Cavanillesia chicamochae), morfología y fenología de la semilla
}

\author{
Evaluation of treatments for propagation of ceiba barrigona (Cavanillesia chicamochae), \\ morphology and phenology of the seed
}

\begin{abstract}
Andrés Mauricio Martínez ${ }^{1 *}$, Yenny Alejandra Ayala ${ }^{1}$, Julián Mauricio Botero ${ }^{1}$, Erika Mayerly Celis ${ }^{1}$, Diego Suescún ${ }^{1}$
\end{abstract}

\begin{abstract}
Resumen
El bosque seco tropical (bs-T) es uno de los ecosistemas más amenazados del planeta, como consecuencia de la presión antrópica. En el enclave subxerofítico del cañón del Chicamocha habita Cavanillesia chicamochae, especie endémica y amenazada que presenta poca supervivencia de sus plántulas, afectando la regeneración de sus poblaciones. El presente estudio evaluó la germinación de $C$. chicamochae con frutos, semillas y estacas, utilizando tres sustratos diferentes. Se emplearon tres tratamientos, los cuales consistieron en diferentes sustratos: T1: arena de río; T2: tierra negra; T3: mezcla 3/4 tierra -1/4 cascarilla de arroz y se utilizaron frutos, semillas desnudas y estacas. Se implementó un diseño completamente aleatorio con tres sustratos incluyendo frutos y semillas y se realizaron cuatro réplicas. Las variables analizadas se sometieron a un ANOVA; cuando las diferencias fueron significativas $(p<0,05)$, además se desarrolló un análisis de medias utilizando la prueba de rangos múltiples de Duncan. El porcentaje de germinación por semilla y fruto presentó diferencias significativas; el tratamiento de mayor eficiencia fue T1 (71\%-semillas y $54 \%$-frutos). Las semillas almacenadas en condiciones ambientales perdieron su viabilidad a partir de los cuatro meses. El mucílago presentó un adecuado valor bromatológico para la alimentación de rumiantes e importantes contenidos en metabolitos secundarios, con usos potenciales en el área farmacéutica, siendo un valor agregado para la especie. El estrés hídrico selecciona características de conservación del agua para garantizar la sobrevivencia de especies en el tiempo, una mejor comprensión de estas estrategias ayudará a predecir respuestas ante futuras sequías.
\end{abstract}

Palabras clave: bosque seco tropical, conservación, Malvaceae, germinación de la semilla, restauración ecológica, servicios ecosistémicos

\begin{abstract}
The tropical dry forest (bs-T) is one of the most threatened ecosystems on the planet, because of anthropic pressure. The subxerophytic enclave of the Chicamocha canyon is inhabited by Cavanillesia chicamochae, an endemic and threatened species that has little survival of its seedlings, affecting the regeneration of its populations. In the present study, the germination of $C$. chicamochae was evaluated with fruits, seeds, and cuttings, using three different substrates. Three treatments were used, which consisted of different substrates: T1: river sand; T2: black earth; T3: $\operatorname{mix} 3 / 4$ soil $-1 / 4$ rice husk and fruits, bare seeds and cuttings were used. A completely randomized design was implemented with three substrates including fruits and seeds and four replicas were performed. The variables analyzed were subjected to an ANOVA, when the differences were significant $(p<0.05)$, in addition an analysis of means was developed using Duncan's multiple range
\end{abstract}

\footnotetext{
1. Instituto de Proyección Regional y Educación a Distancia (IPRED) - Universidad Industrial de Santander, Sede Málaga, Colombia.

* Autor para correspondencia: <ing.martinez8692@gmail.com >
} 
test. The germination percentage per seed and fruit showed significant differences $(p<0.05)$, the treatment with the highest efficiency was T1 (71\%-seeds and 54\%-fruits). Seeds stored under ambient conditions lost their viability after four months. The mucilage presented an adequate bromatological value for the feeding of ruminants and important contents in secondary metabolites, with potential uses in the pharmaceutical area being an added value for the species. Water stress selects water conservation characteristics to guarantee the survival of species over time, a better understanding of these strategies will help predict responses to future droughts.

Keywords: Tropical dry forest, conservation, Malvaceae, seed germination, ecological restoration, ecosystems services

\section{INTRODUCCIÓN}

El bosque seco tropical (bs-T) posee un elevado número de endemismos, formas de vida, grupos funcionales y un alto grado de heterogeneidad de plantas entre regiones geográficas (Linares-Palomino et al., 2011). Se caracteriza por su alto número de especies arbóreas con semillas pequeñas, secas y dispersadas por el viento (Vieira y Scariot, 2006). Este ecosistema es catalogado como uno de los más amenazados (Pizano y García, 2014), como consecuencia de factores globales como el cambio climático y la fragmentación del hábitat (Fajardo et al., 2013), quedando en Colombia solo un $4 \%$ de su cobertura original (HerazoVitola et al., 2017). En el bs-T la inconsistencia en el principio de la estación húmeda y ocurrencia de marcadas estaciones secas, son fuentes importantes de mortalidad por desecación de semillas y plántulas recién germinadas. Esto sumado a que la aridez está aumentando en muchas regiones debido a los aumentos de la temperatura asociados con el cambio climático (Wolfe y Kursar, 2015). Predecir cómo los relictos de bosques secos responderán a las futuras sequías, requiere un conocimiento de los mecanismos por los cuales los árboles tropicales sobreviven a la sequía (Wolfe y Kursar, 2015).

Particularmente, la vegetación del enclave subxerofítico del Cañón del Chicamocha, presenta cambios debido al pastoreo no estabulado de cabras (Capra sp.) y la alta erosión (Valencia et al., 2012). En este cañón habita la ceiba barrigona (Cavanillesia chicamochae Fern. Alonso), especie endémica y amenazada (en peligro-según la Unión Internacional para la Conservación de la Naturaleza-UICN) que crece en altas pendientes sobre suelos jóvenes. Actualmente, solo se observan individuos adultos de la ceiba barrigona
(Rojas, 2014), ya que en la época de reproducción las plántulas son consumidas por las cabras; esto es un problema puesto que la depredación de semillas es una barrera importante para la germinación y regeneración de los ecosistemas (Vieira et al., 2008). Además, la inestabilidad del terreno es otro factor que limita y amenaza la perdurabilidad de las poblaciones de ceiba barrigona en el tiempo (Díaz-Pérez et al., 2011).

En general, la investigación sobre germinación de semillas es limitada (Lu et al., 2016) y la mayoría de estudios abordan determinadas especies como: Crescentia cujete, Platymiscium pinnatum, Bactris guineensis, Brosimum alicastrum, Dilodendron costarricense, Melicoccus bijugatus, Spondias mombin, Albizia saman, Aspidosperma polyneuron, Caesalpinia ebano y Cordia alliodora. La germinación depende de diferentes factores ambientales, además de un conocimiento apropiado de la biología de las semillas y las técnicas de propagación (Bareke, 2018). Por otro lado, la identificación de rasgos funcionales en las semillas ayuda a entender el efecto de disturbios antrópicos reflejados en la dispersión, colonización y establecimiento de plántulas, por lo cual, tales rasgos se asocian con la regeneración natural del ecosistema, contribuyendo no solo a entender la dinámica de las comunidades, sino también a generar pautas para la conservación in situ y ex situ de las especies (Romero, 2016). De esta manera, los rasgos funcionales actúan como indicadores de las estrategias ecológicas de las especies (Lohbeck et al., 2015), las cuales sostienen la dinámica de la comunidad frente a factores antrópicos (Bihn et al., 2010).

A pesar de esto, la mayoría de los estudios en germinación y rasgos funcionales de semillas se enfocan en bosques húmedos (Fajardo et al., 2013; Lohbeck et 
al., 2015). Vieira y Scariot (2006), encontraron cinco veces más estudios sobre la regeneración natural en los bosques tropicales húmedos que en los bosques secos. Particularmente, en Colombia pocos estudios se orientan a la conservación de $C$. chicamochae y hasta ahora el bosque seco empieza a ser estudiado desde su ecología (Fremout et al., 2021; GonzálezM et al., 2018; Thomas et al., 2017), siendo esto un avance importante para orientar procesos de restauración ecológica (Portillo y Sánchez, 2010).

Para lograr una adecuada restauración ecológica, es importante la propagación exitosa de especies arbóreas nativas (Lu et al., 2016), por lo que establecer métodos de propagación eficientes ayudará a determinar metodologías propicias de siembra que aseguren la subsistencia de las poblaciones en el tiempo. El presente estudio tuvo como objetivos $i$ ) evaluar métodos de propagación de $C$. chicamochae para determinar el sustrato más propicio para una mejor eficiencia de germinación de la semilla desnuda, fruto y estacas; ii) evaluar el desarrollo en las primeras etapas de crecimiento; iii) identificar la morfología interna de la semilla, su fenología, su viabilidad al almacenarla bajo condiciones ambientales, y la caracterización fisicoquímica del mucílago.

\section{MATERIALES Y MÉTODOS}

\section{Área de estudio}

El estudio se realizó en la parte baja del Río Guaca $\left(6^{\circ} 41^{\prime} \mathrm{N}\right.$ y $72^{\circ} 54^{\prime} \mathrm{W} ; 6^{\circ} 42^{\prime} \mathrm{N}$ y $\left.72^{\circ} 52^{\prime} \mathrm{W}\right)$, en el sector boquerón, en las veredas La Habana y El Embudo (municipio de Cepitá y San Andrés, respectivamente), Santander. En este sitio se recolectaron las semillas en parches de vegetación subxerofítica. Los análisis de germinación y desarrollo se realizaron en el municipio de Málaga, Santander $\left(6^{\circ} 42^{\prime} \mathrm{N}\right.$ y $72^{\circ} 44^{\prime} \mathrm{W}$ ) a una altitud de $2200 \mathrm{~m} \mathrm{s.} \mathrm{n.} \mathrm{m.} \mathrm{y} \mathrm{una}$ temperatura media de $18^{\circ} \mathrm{C}$ (figura 1 ).

\section{Recolección del material vegetal}

En la temporada seca se ubicaron individuos con fructificación (entre diciembre/2017 y febrero/2018) y con una desjarretadera desinfectada, se recolectaron frutos sanos de color pardo directamente del árbol y se almacenaron en costales de nylon para luego trasladarlos al vivero, donde fueron sembrados al siguiente día. Para las estacas, se seleccionaron ramas verticales con cuatro a seis nudos, realizando cortes de forma oblicua por debajo del nudo en la rama principal. En árboles grandes, se recolectaron estacas con yemas apicales de $20 \mathrm{~cm}$ de longitud y se dejaron entre dos y tres hojas para evitar la deshidratación. Las estacas fueron depositadas en contenedores plásticos y cubiertas con papel periódico húmedo para su traslado al vivero.

\section{Sustratos y tratamientos}

Los tratamientos consistieron en diferentes sustratos: T1: arena de río; T2: tierra negra; T3: mezcla 3/4 tierra y $1 / 4$ cascarilla de arroz. Se sembraron frutos, semillas desnudas (semillas en adelante) y estacas. Para las semillas, de forma manual se retiraron las alas y el mucílago del fruto, con la precaución de no afectar la testa. Posteriormente, para evitar el ataque de hongos a las semillas, se aplicó un fungicida agrícola a base de Tebuconazol (200 g/l) y Trifloxistrobina (100 g/l). Cuando alguna de las plántulas presentó damping off, inmediatamente se retiró de la cama de germinación y se le aplicaron riegos con oxicloruro de cobre. Con los tres sustratos y siembra de frutos y semillas, se implementó un diseño completamente aleatorio; se realizaron cuatro réplicas por tratamiento para un total de 24 unidades experimentales. En cada unidad experimental se establecieron 25 frutos/semillas. La asignación de los tratamientos en las unidades experimentales se realizó de forma aleatoria para garantizar independencia. Para el proceso experimental, se implementaron nueve camas de germinación previamente desinfectadas con oxicloruro de cobre, las semillas se enterraron a $3 \mathrm{~cm}$ de profundidad con una distancia de siembra de $5 \times 7 \mathrm{~cm}$ entre sí. Se realizó un riego inicial, luego riegos cada tres días hasta la segunda semana y posteriormente, cada cinco días. Para las estacas se utilizaron dos tratamientos pregerminativos y un control; el primer tratamiento consistió en sumergir las estacas en ácido gibereleco al $40 \%$ utilizando $1 \mathrm{~g} / \mathrm{l}$ de agua durante 5 min. El segundo, consistió en sumergir las estacas en agua de coco durante $15 \mathrm{~min}$. Se emplearon tres cajones (uno por sustrato, los mismos sustratos de la germinación por frutos y semillas) y se establecieron 
25 estacas por cada tratamiento.

\section{Monitoreo y procesamiento de datos}

El monitoreo de la germinación, la mortalidad y el desarrollo de las plántulas, se realizó a escala diaria, entre el día 0 y el día 105. Para determinar diferencias estadísticas, se realizó un análisis de varianza (ANOVA); cuando las diferencias fueron significativas $(p<0,05)$ se desarrolló un análisis de medias utilizando la prueba de rangos múltiples de Duncan. Todos los análisis se desarrollaron con el programa estadístico R versión 4.0.4 (R Core Team, 2018).

\section{Estadios fenológicos de la semilla}

Se ubicaron algodones en el fondo de diez vasos donde se pusieron semillas en la parte superior hidratándolas periódicamente. También se sumergieron semillas totalmente en diez vasos con agua. Luego, se determinó el estado de geminación, brote y crecimiento de la hoja. Se describió durante 105 días el desarrollo de los estadios fenológicos. Después del trasplante, se implementó la propagación sexual bajo condiciones semi-controladas, en camas de germinación y bolsas de vivero. Los estadios de crecimiento fueron descritos usando la escala de codificación $\mathrm{BBCH}$, basada en un sistema para una codificación uniforme de identificación fenológica de estadios de crecimiento para todas las especies de plantas mono y dicotiledóneas (Bleiholder et al., 1996).
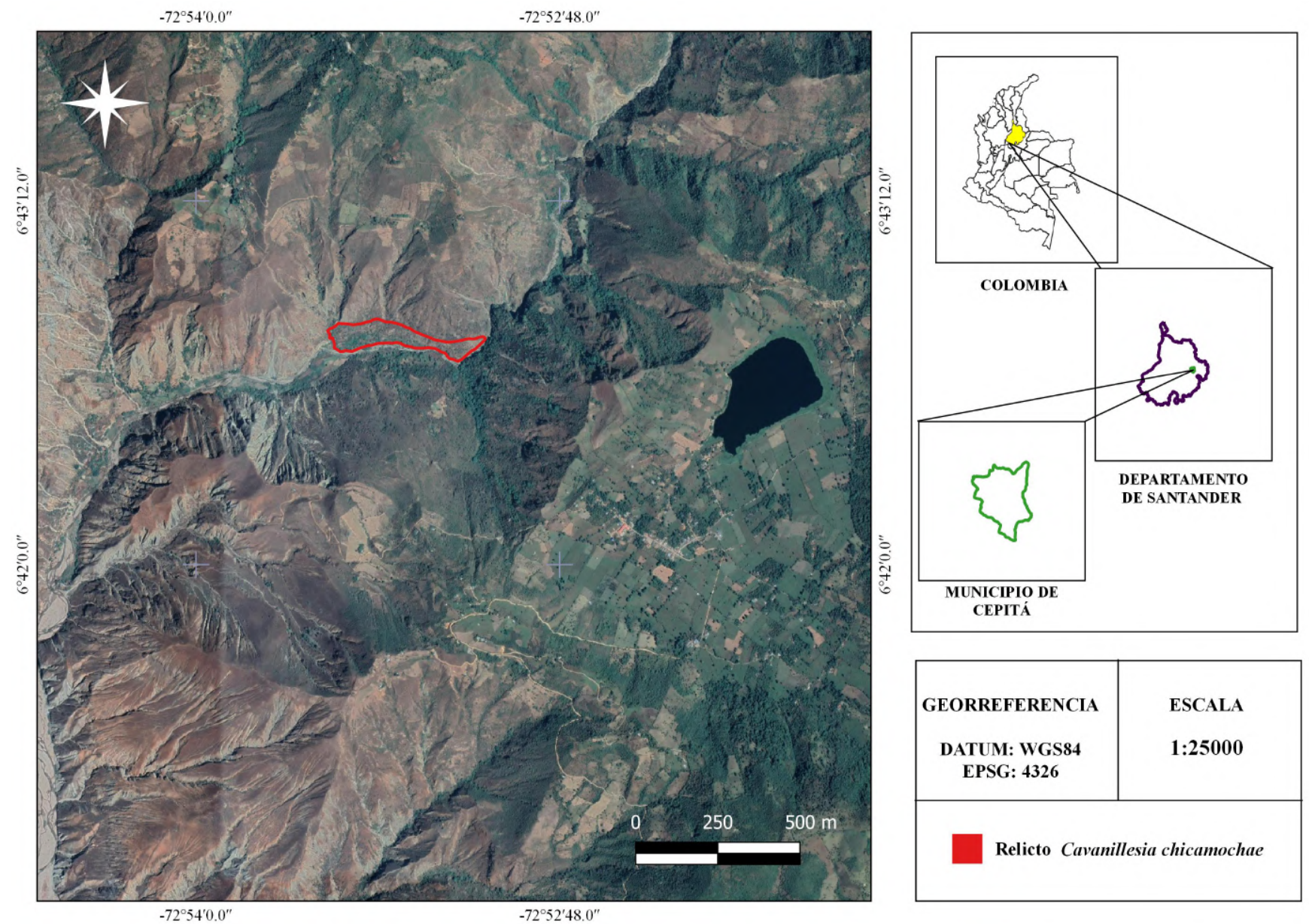

Figura 1. Ubicación de las poblaciones de la ceiba barrigona (Cavanillesia chicamochae) en el área de estudio. 


\section{Morfología, absorción de agua, curva de imbibi- ción y viabilidad de la semilla}

Para determinar la posición, forma y partes del embrión, se realizaron cortes longitudinales de la semilla. Para la curva de imbibición, se registró el peso fresco de diez semillas; éstas se ubicaron en diferentes frascos con $50 \mathrm{ml}$ de agua destilada. En intervalos de $15 \mathrm{~min}$, se registró el peso de cada semilla hasta que el peso de la biomasa no variara en el tiempo (Moreno et al., 2006). Con los datos obtenidos se realizó la curva de imbibición y la masa con respecto al tiempo. Para cuantificar la capacidad de absorción de agua de las semillas, se tomaron diez de éstas y se sumergieron en $50 \mathrm{ml}$ de agua destilada durante $12 \mathrm{~h}$, realizando un pesaje al inicio y al final del ensayo. Finalmente, para determinar la viabilidad de la germinación en el tiempo, se replicaron los ensayos a los dos y cuatro meses; las semillas fueron almacenadas bajo condiciones ambientales.

\section{Análisis bromatológico y de metabolitos secunda- rios del mucílago}

Con la finalidad de identificar las características del mucílago que recubre la semilla, se realizaron análisis bromatológicos y de metabolitos secundarios. Para el contenido de humedad se utilizaron, aproximadamente, 5,6 g de muestra de mucílago a una temperatura de $105^{\circ} \mathrm{C}$ en un horno Memmertö por $24 \mathrm{~h}$; luego del tiempo transcurrido se enfrió en el desecador y se pesó para la determinación de humedad por diferencia de peso. Por otro lado, se determinó el contendió de cenizas tomando muestras en un crisol previamente pesado, se sometió a una temperatura de $550^{\circ} \mathrm{C}$ por seis horas para lo cual se utilizó una mufla. Posteriormente, se determinó el contenido de cenizas por diferencia de pesos. Para el contenido de grasas se implementó un montaje Soxhlet, insertando un cartucho de papel con 7,9 g de mucílago triturado y se utilizaron $160 \mathrm{ml}$ de hexano como solvente sobre una plancha de calentamiento por $8 \mathrm{~h}$. Luego, el cartucho de papel se dejó enfriar por $24 \mathrm{~h}$ para luego montar la muestra en el horno y así evaporar el disolvente completamente hasta obtener el extracto. Para obtener el valor del $\mathrm{pH}$ se diluyeron $3 \mathrm{~g}$ de mucílago en $90 \mathrm{ml}$ de agua destilada, dejando reposar por 10 min para luego realizar la medición con el pHmetro. Estos análisis se realizaron en el Laboratorio de Reconversión Ganadera y Agroforestal de la Universidad Industrial de Santander (UIS), mientras que el resto de los análisis bromatológicos y de metabolitos secundarios del mucílago, se realizaron en el laboratorio de AGROSAVIA.

\section{RESULTADOS}

\section{Germinación, mortalidad, trasplante y viabilidad de la semilla}

Los resultados mostraron un mayor porcentaje de germinación $(71 \%)$ al sembrar semillas en arena de río (T1), con diferencias significativas $(p<0,05)$ entre los sustratos (54\% para frutos y $0 \%$ para estacas) (figura 2I). La pregerminación inició al tercer día con la salida de la radícula, mientras la germinación inició el día nueve finalizando el día 13, determinada en el momento donde se da la extensión completa de los cotiledones. Los factores que influyeron en la germinación de la semilla se presentan en la tabla 1. El primer par de hojas verdaderas se registró a los 45 días de la siembra; en este momento las plántulas se trasplantaron a bolsas de $10 \mathrm{~kg}$ con sustrato de tierra negra, donde se mantuvieron hasta el día 105; para esta fecha, las plántulas presentaron una altura promedio de $20 \mathrm{~cm}$ y una supervivencia del $100 \%$.

Solo una estaca presentó brote de hojas a los 15 días, indicando baja viabilidad por este método. Con respecto al porcentaje de mortalidad en semillas, no se presentaron diferencias significativas $(p<0,05)$ entre los tratamientos T2 $(43,8 \%)$ y $\mathrm{T} 3(49,8 \%)$, aunque ambos tratamientos presentaron diferencias significativas $(p<0,05)$ con T1 $(14,0 \%)$, siendo T1 el tratamiento donde se observó la menor mortalidad (figura 2II).

\section{Codificación BBCH de los estados fenológicos de desarrollo de la semilla}

La germinación de la semilla comenzó con un marcado hinchamiento de la cubierta seminal, la cual está compuesta por mucílago. La radícula rompe el tegumento seminal en uno de los extremos de la semilla y es la primera estructura del embrión que toma contacto con el ambiente exterior al tercer día. 
Posteriormente, el hipocótilo arrastra los cotiledones fuera del tegumento hacia el suelo, donde se desprende de forma horizontal levantando la planta para consecutivamente desplegar los cotiledones. Finalmente, la morfología de la semilla presenta una germinación epigea de tipo "embrión periférico lateral", con una testa blanda en su mayor parte conformada por mucílago y un meristemo radicular opuesto al hilo.

\section{Absorción de agua de la semilla}

El porcentaje de absorción de agua en la semilla fue del $82,8 \%$ partiendo de 1,5 g y alcanzando un promedio de $8,7 \mathrm{~g}$. El fruto, por poseer mayor cantidad de mucílago, presentó una mayor absorción de agua $(91,8 \%)$ (tabla 2$)$.

\section{Curva de imbibición}

En los primeros 15 min se observó un rápido incremento en el peso de la semilla por absorción de agua, el cual pasó de 1,5 a 6,8 g presentando un 78,6\% de imbibición (figura 3). Después de una hora, el peso no presentó variación significativa.

\section{Afectaciones en la germinación}

Hongos: presentaron incidencia en el $24,8 \%$ de las plantas con afectaciones por damping off, tanto en la emergencia de la radícula como en la base de la planta. Se encontraron hongos de los géneros Fusarium, Pythium y Rhizoctonia.

Ausencia en el desprendimiento de la testa de los cotiledones o del hipocótilo: se presentó en el 18,3\%
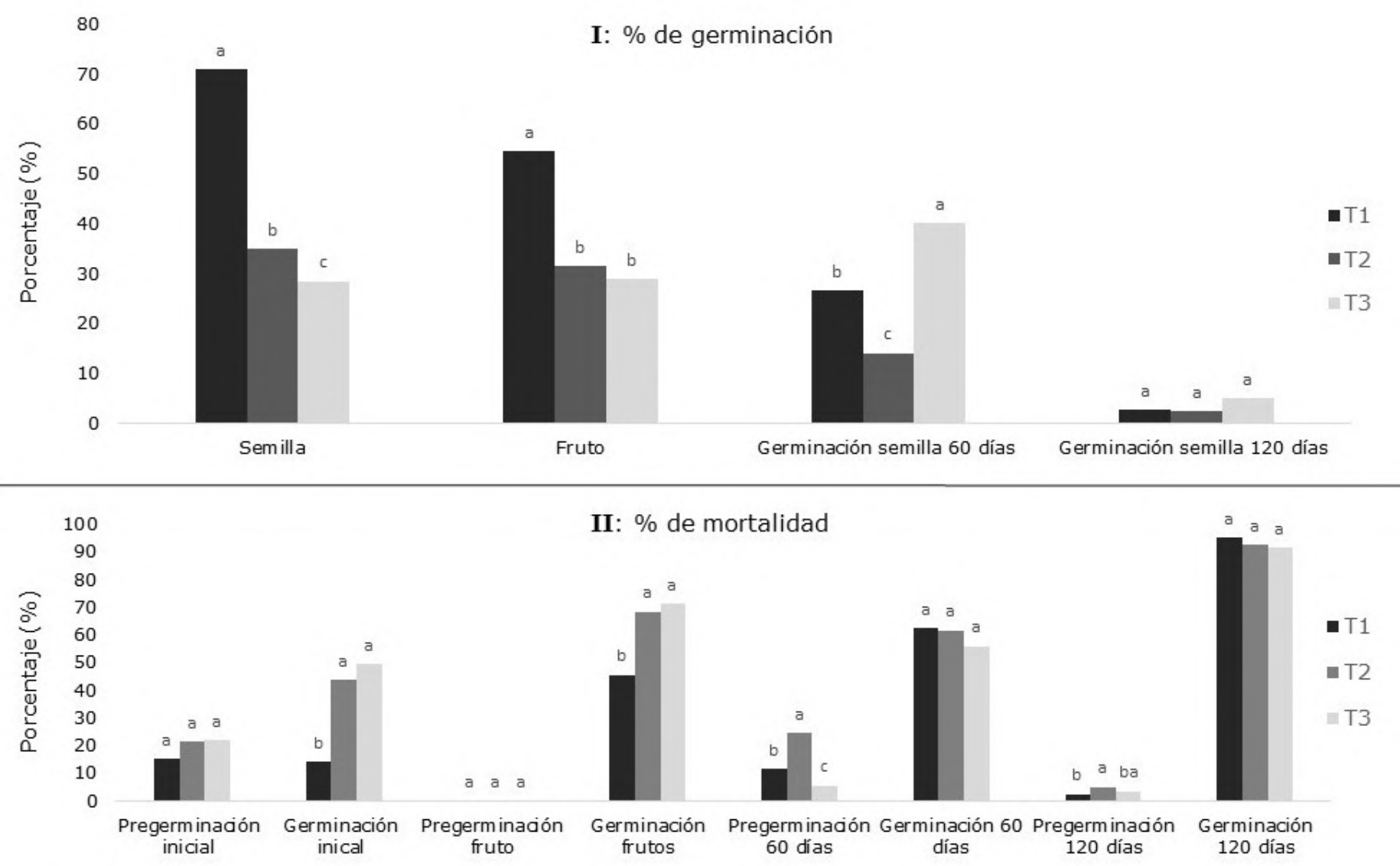

Figura 2. Comparación entre el porcentaje de germinación (I) y mortalidad (II). 
Tabla 1. Descripción de los factores que influyeron en la germinación de la semilla del Cavanillesia chicamochae

\begin{tabular}{|c|c|}
\hline Factores & Descripción \\
\hline Tipo de semilla & $\begin{array}{l}\text { Recalcitrante (conocidas como semillas no ortodoxas). Son semillas que no } \\
\text { sobreviven en condiciones de sequedad y frío; no pueden ser conservadas } \\
\text { por largos periodos porque pueden perder su viabilidad. }\end{array}$ \\
\hline Tipo de germinación & $\begin{array}{l}\text { Epigeas. Los cotiledones emergen del suelo debido a un considerable } \\
\text { crecimiento del hipocotíleo (porción comprendida entre la radícula y el } \\
\text { punto de inserción de los cotiledones). Posteriormente, en los cotiledones } \\
\text { se diferencian cloroplastos, transformándolos en organismos fotosintéticos y } \\
\text { actuando como si fueran hojas }\end{array}$ \\
\hline Impermeabilidad de la semilla & $\begin{array}{l}\text { No impermeable, dado que la testa es de mucílago el cual cumple la } \\
\text { función de absorber y almacenar la humedad para generar un ambiente } \\
\text { óptimo para germinar }\end{array}$ \\
\hline Contenido de agua & Bajo \\
\hline Tolerancia a la deshidratación & Baja \\
\hline Tolerancia a bajas temperaturas & Baja \\
\hline Latencia & $\begin{array}{l}\text { Baja, dado que la viabilidad de la semilla disminuye después del segundo } \\
\text { mes de recolección }\end{array}$ \\
\hline Presencia de luz en la germinación & No es necesaria \\
\hline Presencia de aire en la germinación & No es necesaria \\
\hline Tolerancia a la humedad & Baja \\
\hline
\end{tabular}

de las plantas, caracterizado por su incapacidad para retirarse el tegumento seminal de sus cotiledones o del hipocotíleo, lo que impidió emerger.

Albinismo: se observó en un individuo por ausencia de pigmentos, siendo fatal por la deficiencia de clorofila impidiendo la producción de nutrientes (figura 4).

Poliembrionía: se presentó en $1 \%$ de las plantas; dos embriones se desarrollaron en un solo óvulo o semilla, afectando la germinación (figura 4).

Defoliadores: se presentaron en el 0,5\% de las plantas; los insectos solo dejaron las venas (figura 4).

\section{Análisis bromatológicos y de metabolitos secun- darios del mucílago}

El análisis bromatológico del mucílago evidenció porcentajes de proteína del 10,4\%, energía de 4000 $\mathrm{kcal} / \mathrm{kg}$, digestibilidades superiores al $80 \%$, valores para fibra de detergente neutra (FDN) de 36,9\% y fibra de detergente ácida (FDA) de 17,8\% y contenidos importantes de minerales (tabla 3 ).
Tabla 2. Porcentaje de absorción de agua en la semilla y el fruto

\begin{tabular}{c|c|c|c}
\hline Semilla & $\begin{array}{c}\text { Absorción } \\
\text { de agua } \\
(\%)\end{array}$ & Fruto & $\begin{array}{c}\text { Absorción } \\
\text { de agua } \\
(\%)\end{array}$ \\
\hline 1 & 84,9 & 1 & 93,2 \\
2 & 85,7 & 2 & 92,7 \\
3 & 80,9 & 3 & 93,0 \\
4 & 81,6 & 4 & 90,2 \\
5 & 84,2 & 5 & 92,2 \\
6 & 83,3 & 6 & 91,3 \\
7 & 81,1 & 7 & 92,0 \\
8 & 80,1 & 8 & 90,1 \\
9 & 85,3 & 9 & 91,5 \\
10 & 80,7 & 10 & 91,6 \\
\hline Promedio & 82,8 & Promedio & 91,8 \\
\hline
\end{tabular}

\section{DISCUSIÓN}

La arena de río presentó los mejores resultados de germinación, tanto en semillas como en frutos (figura 4). Esto debido a que es un sustrato infértil y mantiene mayor humedad en las camas de germinación, aportando mejores condiciones y reemplazando las 


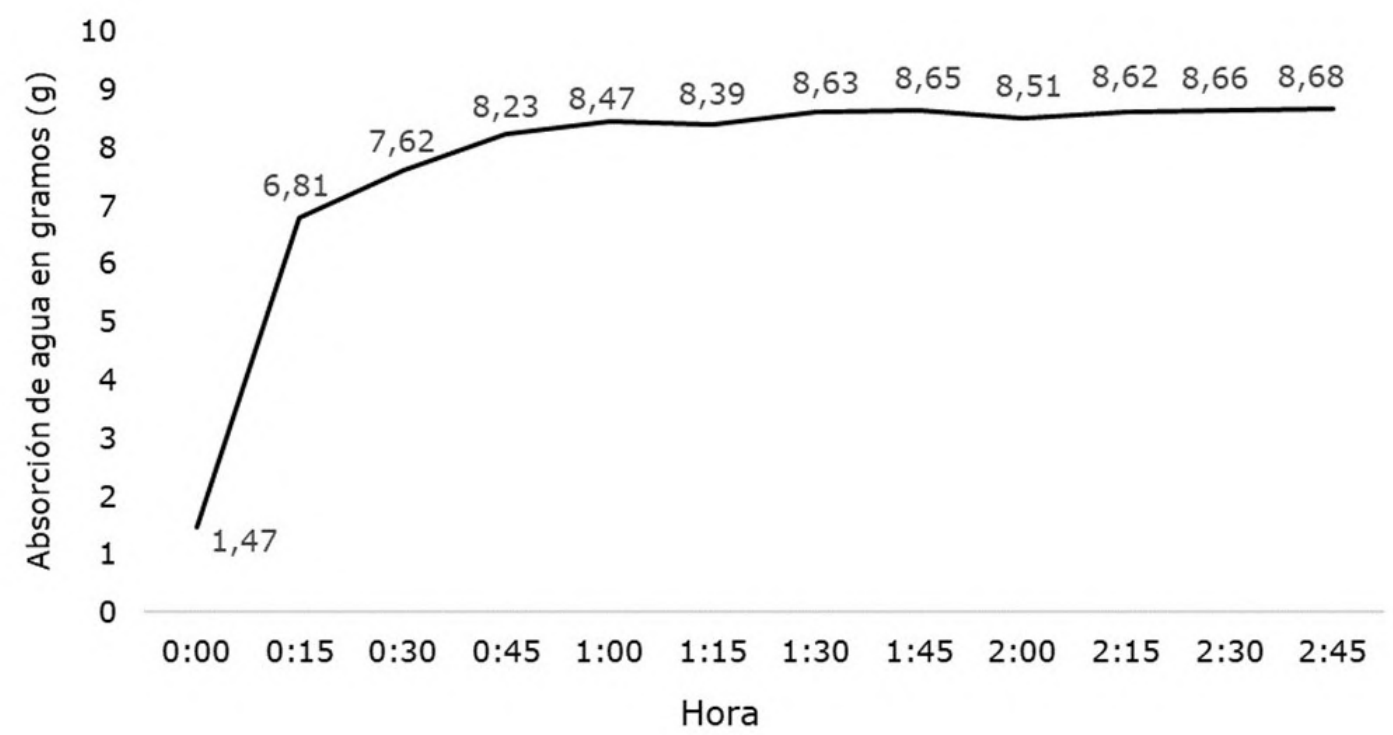

Figura 3. Curva de imbibición de la semilla y aumento en la absorción de agua a través del tiempo.

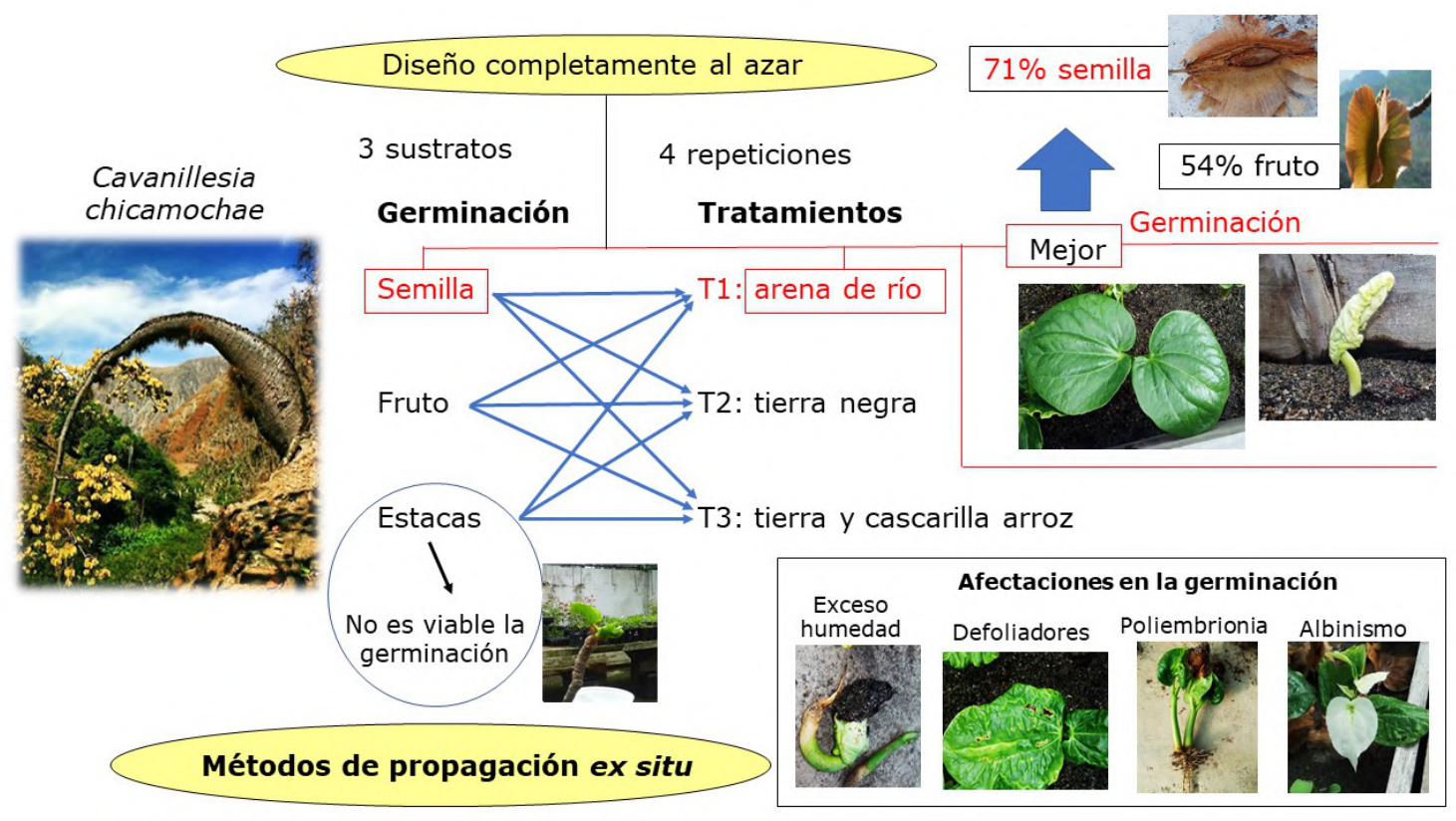

Figura 4. Resumen de los resultados hallados mediante los métodos de propagación ex situ evaluados. 
funciones del mucílago (Kanmegne et al., 2015), mientras que los sustratos de tierra y mezcla presentaron poca humedad y desecación. Resultados similares reportó Garwood (1985), donde evaluó el porcentaje de germinación con otra especie del mismo género (Cavanillesia platanifolia), llegando a la conclusión de que los tratamientos utilizados, entre ellos frutos y semillas desnudas, no tuvieron ningún efecto en la germinación.

Tabla 3. Análisis bromatológico y de metabolitos secundarios del mucílago del Cavanillesia chicamochae

\begin{tabular}{|c|c|c|}
\hline \multicolumn{3}{|c|}{ Análisis bromatológico } \\
\hline Ítem & Unidad & Valor \\
\hline Materia seca total & \multirow{11}{*}{$\%$} & 27,57 \\
\hline Proteína cruda & & 10,38 \\
\hline Ceniza & & 4,39 \\
\hline Extracto etéreo & & 1,30 \\
\hline FDN & & 36,92 \\
\hline FDA & & 17,83 \\
\hline Lignina & & 7,94 \\
\hline Hemicelulosa & & 19,09 \\
\hline Almidón total & & 0,62 \\
\hline NDT & & 75,17 \\
\hline Digestibilidad & & 81,97 \\
\hline Energía bruta & Kcal.kg-1 MS & 4000 \\
\hline Calcio & \multirow{6}{*}{$\%$} & 1,07 \\
\hline Fósforo & & 0,09 \\
\hline Magnesio & & 0,31 \\
\hline Potasio & & 0,64 \\
\hline Sodio & & 0,02 \\
\hline Azufre & & 0,03 \\
\hline Cobre & \multirow{5}{*}{$\mathrm{mg} \cdot \mathrm{kg}-1$} & 9,23 \\
\hline Hierro & & 6,89 \\
\hline Magnesio & & 25,67 \\
\hline Zinc & & 11,1 \\
\hline Boro & & 29,52 \\
\hline \multicolumn{3}{|c|}{ Análisis de los metabolitos secundarios } \\
\hline Ítem & Unidad & Valor \\
\hline Fenoles totales & \multirow{4}{*}{$\%$} & 2,74 \\
\hline Taninos totales & & 2,18 \\
\hline Alcaloides totales & & 0,26 \\
\hline Saponinas & & 4,8 \\
\hline
\end{tabular}

Las condiciones y tiempo de almacenamiento de las semillas pueden ser factores importantes en los procesos de germinación de especies arbóreas (Geovo et al., 2021). A pesar de que Vieira et al. (2008) reportaron que la mayoría de las especies de árboles del bosque seco tienen una alta longevidad de semillas y que se pueden almacenar con éxito a temperaturas naturales, los resultados de viabilidad de las semillas del C. chicamochae muestran que, a partir de los dos meses de recolección, el porcentaje de germinación disminuye en un 26,8\% y pasados cuatro meses al 3,3\%, por esto no se deben almacenar las semillas por mucho tiempo. Al germinar por semilla, se facilita el montaje de los ensayos, el transporte, almacenamiento y control de hongos con la aplicación de fungicidas. Además, el mucílago se retira en el lugar de recolección de la semilla y sirve como abono para el suelo. Plantar las semillas después de que haya pasado la estación seca sería particularmente importante para la restauración de áreas abiertas, donde la desecación de semillas y plántulas es más severa (Vieira et al., 2008).

La germinación de las semillas, al inicio de la estación húmeda, parece ser un rasgo evolutivo en ecosistemas secos, que maximiza el uso de las primeras lluvias para el establecimiento de plántulas, aumentando potencialmente la probabilidad de sobrevivir a la próxima estación seca (Vieira et al., 2008). Las especies de árboles en bosques secos tropicales varían en sus mecanismos de supervivencia a la sequía, por lo que la dependencia de agua almacenada (sea en el tallo o mucílago) se considera una característica clave para la adaptación al cambio climático (Wolfe y Kursar, 2015). Tanto el fruto como la semilla presentaron una alta capacidad para retener agua (91,8 y $82,8 \%$, respectivamente), siendo mayor en el fruto debido al mucílago que actúa como medio natural de almacenamiento de agua para la germinación de la semilla bajo condiciones de sequía típica del Cañón del Chicamocha. Esta reserva de agua le permite a la plántula contar con un ambiente húmedo mientras que la radícula se fija al suelo (Díaz-Pérez et al., 2011). Ya se ha documentado que los mucílagos externos ayudan en la absorción y retención de agua durante la germinación en algunas especies, particularmente en hábitats secos. En otro estudio, la presencia del mucílago disminuyó el grado 
de marchitamiento de las plántulas del Cavanillesia platanifolia y el mucílago permite que las semillas de esta especie germinen rápidamente al comienzo de la temporada de lluvias, antes que otros competidores potenciales (Garwood, 1985).

Los resultados muestran un 78,6\% de imbibición, lo que demuestra un incremento de tamaño por hinchazón debido al mucílago que cubre la semilla, activándose al entrar en contacto con el agua, razón por la cual ésta presenta una rápida y alta germinación (Reino et al., 2008). La razón por la que el peso no presentó una variación significativa después de una hora fue porque el mucílago se desprende con el agua, entrando la semilla en la segunda fase denominada "activación del metabolismo"; en esta fase se distribuye el agua y se movilizan las enzimas encargadas de la activación del embrión, para posteriormente absorber nuevamente agua y entrar a la tercera fase encargada de la elongación del embrión y ruptura de la testa (Bleiholder et al., 1996).

El análisis bromatológico del mucílago de $C$. chicamochae evidenció importantes contenidos de minerales, demostrando su potencial para ser utilizado en modelos de alimentación de rumiantes (Patiño et al., 2011). Los resultados arrojaron valores promedio de 2,7 para fenoles, 2,2 para taninos y $0,3 \%$ para alcaloides, valores que se encuentran dentro del rango de otros estudios. García y Medina (2006), evaluaron metabolitos secundarios en diez especies forrajeras y reportaron valores para fenoles entre 2,2 y $5,7 \%$, taninos entre 0,5 y $5,3 \%$, alcaloides entre 0,04 y $0,51 \%$, y saponinas entre 1,3 y 3,9\%; particularmente, en el mucílago del C. chicamochae encontramos valores de saponinas muy superiores $(4,8 \%)$.

Ortega et al. (2015), en gramíneas de clima cálido (Brachiaria brizantha y Panicum maximum) registraron valores de proteína entre 7,7 y $10,7 \%$, rango que incluye los valores registrados en este trabajo $(10,4 \%)$. Por otro lado, Aye (2016), en tres plantas forrajeras, reportó valores de energía $(\mathrm{kcal} / \mathrm{kg})$ de 3764 (Moringa oleifera), 3755 (Gmelina arborea) y 3912 (Tithonia diversifolia), valores inferiores a los encontrados en el mucílago del C. chicamochae (4000 $\mathrm{kcal} / \mathrm{kg}$ ). Pérez-Almario et al. (2013), en diez especies con potencial forrajero, encontraron valores para
FDN entre 35,4 y $57,9 \%$ y FDA entre 31,2 y $44,1 \%$, lo cual muestra que el mucílago del $C$. chicamochae presenta una baja concentración en fibras estructurales frente a las plantas forrajeras comúnmente utilizadas en la alimentación de rumiantes (36,9 y 17,8\% en FDN y FDA, respectivamente). Rodríguez et al. (2014), en forrajes, registraron valores de digestibilidad del 52,4\% (Moringa olifeira), 79,8\% (Morus alba), 37,2\% (Trichanthera gigantea) y 52,0\% (Leucaena leucocephala). Para dichos forrajes, el mucílago del $C$. chicamochae presentó valores superiores con un $82,0 \%$, esto demuestra el potencial del mucílago en sistemas de alimentación de rumiantes.

Como se puede observar, la físicoquímica del mucílago podría ser potencialmente importante como Producto Forestal No Maderable (PFNM), generando un valor agregado a la especie. Como la ceiba barrigona, gran parte de las especies endémicas se distribuyen en ecosistemas específicos afectados por procesos de cambio ambiental global, presentando un alto grado de peligro de extinción (Sánchez, 2002). Por lo tanto, alternativas de uso potencial podrían ayudar a disminuir la presión sobre la ceiba barrigona, pues su extinción podría alterar la provisión de servicios ecosistémicos asociados a la biodiversidad. Actualmente, la conservación y restauración del bs$\mathrm{T}$ es prioritaria, no solo por su rápida degradación, sino también por su alto número de endemismos y diversidad beta (Fajardo et al., 2013).

En conclusión, el conocimiento integrado de la recolección de semillas, su almacenamiento, y factores y rendimiento de la germinación es vital para los planes de conservación de la biodiversidad específica de cada región (Lu et al., 2016); los resultados encontrados en este estudio contribuirán a mejorar los planes de conservación de la ceiba barrigona y la restauración de su hábitat dentro del Cañón del Chicamocha (Khurana y Singh, 2001). Finalmente, se recomienda: $i$ ) estandarizar un protocolo de riego mediante ensayos de déficit hídrico, lo que permitirá disminuir la presencia de hongos generados por exceso de humedad en semilla y fruto; ii) recolectar las semillas al final de la estación seca y plantarlas al comienzo de la estación húmeda, esto para mejorar el establecimiento de las plántulas y reducir el tiempo de exposición a los depredadores (Vieira et 
al., 2008); iii) aprovechar los nutrientes energéticos y proteicos del mucílago, en proyectos alternativos como sistemas silvopastoriles, elaboración de productos naturales y en el área farmacéutica.

\section{AGRADECIMIENTOS}

Los autores expresan su agradecimiento al Laboratorio de Reconversión Ganadera y Agroforestal, de la Universidad Industrial de Santander, sede Málaga.

\section{CONFLICTO DE INTERESES}

Los autores declaramos que no presentamos ningún conflicto de intereses.

\section{REFERENCIAS}

Aye, P. A. (2016). Comparative nutritive value of Moringa oleifera, Tithonia diversifolia and Gmelina arborea leaf meals. American Journal of Food and Nutrition, 6(1), 2332. 10.5251/ajfn.2016.6.1.23.32

Bareke, T. (2018). Biology of seed development and germination physiology. Advances in Plants $\&$ Agriculture Research, 8(4), 336-46. 10.15406/apar.2018.08.00335

Bihn, J. H., Gebauer, G., \& Brandl, R. (2010). Loss of functional diversity of ant assemblages in secondary tropical forests. Ecology, 91 (3), 782-792. 10.1890/08-1276.1

Bleiholder, H., Buhr, L., Feller, C., Hack, H., Hess, M., Klose, R., Meier, U., Stauss, R., \& Weber, E. (1996). Compendio para la identificación de los estadios fenológicos de especies mono-y dicotiledóneas cultivadas: escala $B B C H$ extendida. Centro Federal de Investigaciones Biológicas para Agricultura y Silvicultura (BBA). https://www.agro.basf.es/Documents/es_ files/pdf_1_files/services_files/descarga.pdf

Díaz-Pérez, C., Puerto, M. A., \& Fernández, J. L. (2011). Evaluación del hábitat, las poblaciones y el estatus de conservación del barrigón (Cavanillesia chicamochae, Malvaceae-Bombacoideae). Caldasia, 33(1), 105119. https://revistas.unal.edu.co/index.php/cal/article/ view $/ 36379 / 37970$

Fajardo, L., Rodríguez, J. P., González, V., \& Briceño-Linares, J. M. (2013). Restoration of a degraded tropical dry forest in Macanao, Venezuela. Journal of Arid Environments, 88, 236-243. 10.1016/j.jaridenv.2012.08.009

Fremout, T., Thomas, E., Bocanegra-González, K. T., AguirreMorales, C. A., Morillo-Paz, A. T., Atkinson, R., AlcazarCaicedo, C., Kettle, C., Gonzalez, R., Gonzalez, M. A., Gutierrez, J. P., Gil-Tobon, C., Moscoso-Higuita, L. G., Lopez-Lavealle, L. L. B., de Carvalho, D., \& Muys, B. (2021). Dynamic seed zones to guide climate-smart seed sourcing for tropical dry forest restoration in Colombia. Forest Ecology and Management, 490, 119127. 10.1016/j.foreco.2021.119127

García, D. E., \& Medina, M. G. (2006). Composición química, metabolitos secundarios, valor nutritivo y aceptabilidad relativa de diez árboles forrajeros. Zootecnia Tropical, 24(3), 233-250. http://ve.scielo.org/scielo.php? script $=$ sci_arttext\&pid $=$ S0798-72692006000300004

Garwood, N. C. (1985). The role of mucilage in the germination of cuipo, Cavanillesia platanifolia (H. \& B.) HBK (Bombacaceae), a tropical tree. American Journal of Botany, 72 (7), 1095-1105. https://www.jstor.org/stable/2443455

Geovo, V. R., Tovar, L. C., Mosquera, P. L. R., Arroyo, J. H. C., \& Ramos, P. A. (2021). Métodos de escarificación química y sus efectos en la germinación de semillas de Ochroma pyramidale Cav. ex Lam. Urb. Revista de Investigación Agraria y Ambiental, 12(1), 8. 10.22490/21456453.3727

González-M, R., García, H., Isaacs, P., Cuadros, H., LópezCamacho, R., Rodríguez, N., \& Pizano, C. (2018). Disentangling the environmental heterogeneity, floristic distinctiveness and current threats of tropical dry forests in Colombia. Environmental Research Letters, 13(4), 045007. 10.1088/1748-9326/aaad74

Herazo-Vitola, F., Mercado Gómez, J., \& Mendoza Cifuentes, H. (2017). Estructura y composición florística del bosque seco tropical en los Montes de María (Sucre-Colombia). Ciencia en Desarrollo, 8(1), 71-82. http://www.scielo. org.co/pdf/cide/v8n1/0121-7488-cide-8-01-00071.pdf

Kanmegne, G., Anouma, M., Fotso, A., Mbouobda, H. D., Mbibong, D. A., \& Omokolo, D. N. (2015). Germination of Cola anomala (K. Shum.) Shott and Endl seeds: effects of provenance, substrate and dehydration. International Journal of Biological and Chemical Sciences, 9(3), 11711180. 10.4314/ijbcs.v9i3.3

Khurana, E. K., \& Singh, J. S. (2001). Ecology of seed and seedling growth for conservation and restoration of tropical dry forest: a review. Environmental Conservation, 28(1), 39-52. 10.1017/S0376892901000042

Linares-Palomino, R., Oliveira-Filho, A. T., \& Pennington, R. T. (2011). Neotropical seasonally dry forests: diversity, endemism, and biogeography of woody plants. En R. Dirzo, H. S., Young, H. A., Mooney, G. Ceballos, Seasonally dry tropical forests (p. 3-21). Island Press.

Lohbeck, M., Lebrija, E., Martínez, M., Meave, A., Poorter, L., \& Bongers, F. (2015). Functional trait strategies of trees in dry and wet tropical forests are similar but differ in their consequences for succession. Plos One, 10(4), e0123741. doi: 10.1371/journal.pone.0123741

Lu, Y., Ranjitkar, S., Xu, J. C., Ou, X. K., Zhou, Y. Z., Ye, J. F., \& He, J. (2016). Propagation of native tree species to restore subtropical evergreen broad-leaved forests in SW China. Forests, 7(1), 12. 10.3390/f7010012

Moreno, F., Plaza, G. A., \& Magnitskiy, S. V. (2006). Efecto de la testa sobre la germinación de semillas de caucho (Hevea brasiliensis Muell.). Agronomía Colombiana, 24 (2), 290-295. https://revistas.unal.edu.co/index.php/ agrocol/article/view/20041

Ortega, C. A., Lemus, C., Bugarin, J. O., Alejo, G., Ramos, A., Grageola, O., \& Bonilla, J. A. (2015). Características agronómicas, composición bromatológica, digestibilidad y consumo animal en cuatro especies de pastos de los géneros Brachiaria y Panicum. Tropical and Subtropical 
Agroecosystems, 18, 291-301. https://www.redalyc.org/ articulo.oa?id $=93944043005$

Patiño, R., Da Silva Filho, J., \& Pérez, J. (2011). Modelos de predicción de exigencias minerales para rumiantes. Revista Colombiana de Ciencia Animal-RECIA, 3(2), 344-365. 10.24188/recia.v3.n2.2011.409

Pérez-Almario, N., Ibrahim, M. V., Skarpe, C., \& Guerin, H. (2013). Diversidad forrajera tropical 1. Selección y uso de leñosas forrajeras en sistemas de alimentación ganadera para zonas secas de Nicaragua. Agroforestería en las Américas, 50, 37-43. http://bco.catie.ac.cr:8087/ portal-revistas/index.php/AGRO/article/view/55

Pizano, C., \& García, H. (Eds.). (2014). Bosques secos tropicales en Colombia. Instituto de Investigaciones de Recursos Biológicos "Alexander von Humboldt" - Ministerio de Ambiente y Desarrollo Sostenible. http://www.humboldt. org.co/images/documentos/pdf/investigacion/ gonzalez-m-et-al-2014-memoria-tecnica-verificacion.pdf

Portillo, C., \& Sánchez, A. (2010). Extent and conservation of tropical dry forests in the Americas. Biological Conservation, 143(1), 144-155. 10.1016/j.biocon.2009.09.020

R Core Team. (2018, Septiembre 20). R: A language and environment for statistical computing. R Foundation for Statistical Computing, Vienna, Austria. Recuperado de https://www.R-project.org/.

Reino, J., González, Y., \& Sánchez, J. A. (2008). Temperatura óptima de germinación y patrones de imbibición de las semillas de Albizia lebbeck, Gliricidia sepium y Bauhinia purpurea. Pastos y Forrajes, 31(3), 1-1. http://scielo.sld.cu/scielo.php?script=sci_arttext\& pid $=$ S0864-03942008000300002

Rodríguez, R., González, N., Alonso, J., Domínguez, M., \& Sarduy, L. (2014). Valor nutritivo de harinas de follaje de cuatro especies arbóreas tropicales para rumiantes. Revista Cubana de Ciencia Agrícola, 48(4), 371. https: //www.redalyc.org/pdf/1930/193033033011.pdf

Rojas, A. (2014). Producción de plántulas de Cavanillesia chicamochae especie en peligro de extinción. Corporación Autónoma para la Defensa de la Meseta de Bucaramanga (CDMB) y Jardín Botánico Eloy Valenzuela. http://www.cdmb.gov.co/web/Link/ PRODUCCIONDEPLANTULASDECavanillesiachicmochae. pdf

Romero, J. (2016). Caracterización morfofisiológica de semillas de especies leñosas distribuidas en dos zonas secas presentes en el Sur del Ecuador. Revista Ecosistemas, 25(2): 93-100. 10.7818/ECOS.2016.25-2.12

Sánchez, E. (2002). Impactos sobre los ecosistemas. En: F. L. Repetto, \& C.S. Karez (Eds.), Notas de clase dictadas en el II Curso Internacional de Aspectos Geológicos de Protección ambiental (313-321). Oficina Regional de Ciencia de la UNESCO para América Latina y el Caribe. http://www.ingenieroambiental.com/4012/ campinasprimeras.pdf

Thomas, E., Alcazar, C., Moscoso H. L. G., Osorio, L. F., Salgado-Negret, B., Gonzalez, M., Parra, M., Bozzano, M., Loo, J., Jalonen, R., \& Ramirez, W. (2017). The importance of species selection and seed sourcing in forest restoration for enhancing adaptive potential to climate change: Colombian tropical dry forest as a model. En: L. Rodríguez, \& I. Anderson (Eds.), The Lima Declaration on Biodiversity and Climate Change: Contributions from Science to Policy for Sustainable Development (p. 122134). Technical Series No.89. Secretariat of the Convention on Biological Diversity. https://cgspace.cgiar.org/ handle/10568/90681

Valencia-Duarte, J., Ortiz, L. N. T., \& Ríos, O. V. (2012). Dinámica de la vegetación en un enclave semiárido del río Chicamocha, Colombia. Biota Colombiana, 13(2) 40-65. http://revistas.humboldt.org.co/index. $\mathrm{php} /$ biota/article/view/262

Vieira, D. L., \& Scariot, A. (2006). Principles of natural regeneration of tropical dry forests for restoration. Restoration Ecology, 14(1), 11-20. 10.1111/j.1526-100X.2006.00100.x

Vieira, D. L. M., de Lima, V. V., Sevilha, A. C., \& Scariot, A. (2008). Consequences of dry-season seed dispersal on seedling establishment of dry forest trees: Should we store seeds until the rains? Forest Ecology and Management, 256(3), 471-481. 10.1016/j.foreco.2008.04.052

Wolfe, B. T., \& Kursar, T. A. (2015). Diverse patterns of stored water use among saplings in seasonally dry tropical forests. Oecologia, 179(4), 925-936. 10.1007/s00442-015$3329-\mathrm{z}$ 